\title{
ESTIMATES FOR WEAK-TYPE OPERATORS
}

\author{
BY COLIN BENNETT
}

\author{
Communicated by E. M. Stein, February 16, 1973
}

1. Introduction. When $f$ is an integrable function on the interval $[0,1]$, we denote by $f^{*}$ its nonincreasing rearrangement and by $f^{* *}$ the average $f^{* *}(t)=(1 / t) \int_{0}^{t} f^{*}(s) d s$. The Lorentz space $L^{p q}, 1 \leqq p \leqq \infty$, $1 \leqq q \leqq \infty$ consists of all functions $f$ for which the norm

$$
\|f\|=\left\{\int_{0}^{1}\left[t^{1 / p} f^{* *}(t)\right]^{q} \frac{d t}{t}\right\}^{1 / q}
$$

is finite; the Lorentz space $L^{(p q)}$ is defined in the same way except that $f^{* *}$ is replaced by $f^{*}$. When $1<p \leqq \infty, L^{p q}$ and $L^{(p q)}$ coincide, up to equivalence of (quasi) norms (cf. [3], [4]). The spaces $L^{p q}, 1<p<\infty$, are the intermediate spaces $\left(L^{1}, L^{\infty}\right)_{\theta, q ; K}, \theta=1-1 / p$, generated by the $K$-method of J. Peetre (cf. [2], [5], [7]). Note that $L^{(1 \infty)}$ is the space usually referred to as "weak- $L^{1}$ " and that the Orlicz space $L \log ^{+} L$ of functions $f$ for which $|f| \log ^{+}|f|$ is integrable, is (cf. [1]) none other than the Lorentz space $L^{11}$. Thus

$$
L \log ^{+} L=L^{11} \subseteq L^{1 \infty}=L^{1}=L^{(11)} \subseteq L^{(1 \infty)}=\text { weak- } L^{1} .
$$

From characterizations of the intermediate spaces $\left(L \log ^{+} L, L^{1}\right)_{\theta, q ; K}$ and $\left(L^{1} \text {, weak }-L^{1}\right)_{\theta, q ; K}$ obtained by the author in [1] and subsequently, there follow some new estimates for weak-type operators. In particular, we obtain a sharper form of a theorem of O'Neil [6] concerning operators that are simultaneously of weak-types $(1,1)$ and $(p, p), 1<p \leqq \infty$.

2. Intermediate spaces between $L \log ^{+} L$ and $L^{1}$. The space of functions $f$ for which the norm

$$
\|f\|=\left\{\int_{0}^{1}\left[t\left(\log \frac{1}{t}\right)^{\theta-1 / q} f^{* *}(t)\right]^{q} \frac{d t}{t}\right\}^{1 / q}
$$

is finite, will be denoted by $A^{\theta q}, 0<\theta<1,1 \leqq q \leqq \infty$. The corresponding space with $f^{* *}$ replaced by $f^{*}$ in the previous definition, is denoted by $A^{(\theta q)}$. The following results were obtained by the author in [1]:

TheOREM 1. $\left(L^{1}, L \log ^{+} L\right)_{\theta, q ; K}=A^{\theta q}, 0<\theta<1,1 \leqq q \leqq \infty$.

Corollary 1.1. $\left(L^{1}, L \log ^{+} L\right)_{\theta, 1 ; K}=L\left(\log ^{+} L\right)^{\theta}, 0<\theta<1$.

AMS (MOS) subject classifications (1970). Primary 46E30, 46E35. 
CoROllary 1.2. $\left(L^{1}, L \log ^{+} L\right)_{1 / q, q ; K}=L^{1 q}, 1<q<\infty$.

Also characterized in [1] are the spaces $\left(L \log ^{+} L, L^{\infty}\right)_{\theta, q ; K}$. We have no need here of any explicit characterization but let us note the following result.

THEOREM 2. $\left(L \log ^{+} L, L^{\infty}\right)_{\theta, q ; K} \subsetneq L^{p q}, \theta=1-1 / p$.

The proofs of all these results depend crucially on the fact that the Orlicz space $L \log ^{+} L$ is also a Lorentz $\Lambda$-space (cf. [1]). In the next section when we consider the space weak- $L^{1}$, the situation is radically different and the same techniques do not apply.

3. Intermediate spaces between $L^{1}$ and weak $-L^{1}$. For a measurable function $f$ on $[0,1], f^{\#}$ denotes the nonincreasing rearrangement on $(0, \infty)$ of the function $t f^{*}(t)$, taken with respect to the measure $d m^{*}(t)=d t / t$. Thus $f^{\#}$ is the right-continuous inverse of the distribution function $\sigma \rightarrow m^{*}\left\{t: t f^{*}(t)>\sigma\right\}$. The next theorem shows that the intermediate spaces between $L^{1}$ and weak- $L^{1}$ are simply the "Lorentz spaces with respect to $f^{\#,}$.

THEOREM 3. A necessary and sufficient condition that $f$ belong to $\left(L^{1} \text {, weak- } L^{1}\right)_{\theta, q ; K}, 0<\theta<1,1 \leqq q \leqq \infty$, is that the quasinorm

be finite.

$$
\|f\|=\left\{\int_{0}^{\infty}\left[t^{1-\theta} f^{\#}(t)\right]^{q} \frac{d t}{t}\right\}^{1 / q}
$$

It is not difficult to check that the quasinorm in the statement of Theorem 3 dominates the quasinorm of the space $A^{(1-\theta, q)}$. Thus

Corollary 3.1. $\left(L^{1} \text {, weak- } L^{1}\right)_{\theta, q ; K} \subseteq A^{(1-\theta, q)}$.

The Peetre $K$-functional norm $K(t ; f)=K\left(t ; f\right.$; weak- $\left.L^{1}, L^{\infty}\right)$ (cf. [2], [5], [8]) for the pair (weak- $\left.L^{1}, L^{\infty}\right)$ is given by $K(t ; f)=\sup _{0<s<t} s f^{*}(s)$. Since $f^{*}(t) \leqq t^{-1} K(t ; f) \leqq f^{* *}(t)$, it follows that

$$
L^{p q} \subseteq\left(\text { weak- } L^{1}, L^{\infty}\right)_{\theta, q ; K} \subseteq L^{(p q)},
$$

$\theta=1-1 / p$. Hence

THEOREM 4. (weak- $\left.L^{1}, L^{\infty}\right)_{\theta, q ; K}=L^{p q}, \theta=1-1 / p, 1<p<\infty, 1 \leqq$ $q \leqq \infty$.

4. The interpolation theorems. We are now in a position to exhibit the interpolation theorems corresponding to the various classes of intermediate spaces described in $\S \S 2$ and 3. Our main result is:

THEOREM 5. Let $T$ be a quasilinear (cf. [5]) operator, $T: L \log ^{+} L \rightarrow L^{1}$ and $T: L^{1} \rightarrow$ weak- $L^{1}$. Then $T: A^{\theta q} \rightarrow A^{(\theta q)}, 0<\theta<1,1 \leqq q \leqq \infty$. 
The cases $\theta=1 / q$ and $q=1$ yield the following corollaries:

COROLlaRY 5.1. Under the hypotheses of Theorem 5, $T$ is a bounded operator from the Lorentz space $L^{1 q}$ into the Lorentz space $L^{(1 q)}$, $1 \leqq q \leqq \infty$.

COROLlaRY 5.2. Under the hypotheses of Theorem 5, $T$ is a bounded operator from the Orlicz space $L\left(\log ^{+} L\right)^{\theta}$ into the space $A^{(\theta 1)}, 0<\theta<1$.

Any operator $T$ of weak-types $(1,1)$ and $(p, p), p>1$, will satisfy the hypotheses of Theorem 5 (cf. [6]) and hence the conclusions above; in particular, any such operator maps $L\left(\log ^{+} L\right)^{\theta}$ into $A^{(\theta 1)}$. Since $A^{(\theta 1)} \varsubsetneqq$ $A^{(\theta, 1 / \theta)}=L^{(1,1 / \theta)}$, Corollary 5.2 is sharper than the following result of O'Neil [6]:

Corollary 5.3 (O'NeIL). If $T$ is of weak-types $(1,1)$ and $(p, p), p>1$, then $T: L\left(\log ^{+} L\right)^{\theta} \rightarrow L^{(1,1 / \theta)}$.

Finally, let us note that if we combine Theorem 4 and the fact that the $L^{p q}$ spaces are the intermediate spaces between $L^{1}$ and $L^{\infty}$, we can reproduce the following special case of the Marcinkiewicz-Calderón-Hunt theorem (cf. $[3],[4],[5],[8]$ ).

THEOREM 6 (MARCINKIEWICZ-CALDERóN-HUNT). If $T$ is of weak-types $(1,1)$ and $(\infty, \infty)$, then $T: L^{p q} \rightarrow L^{p q}, 1<p<\infty, 1 \leqq q \leqq \propto$.

\section{REFERENCES}

1. C. Bennett, Intermediate spaces and the class $L \log ^{+} L$, Ark. Mat. (to appear).

2. P. L. Butzer and H. Berens, Semi-groups of operators and approximation, Die Grundlehren der math. Wissenschaften, Band 145, Springer-Verlag, New York, 1967. MR 37 \#5588.

3. A. P. Calderón, Spaces between $L^{1}$ and $L^{\infty}$ and the theorem of Marcinkiewicz, Studia Math. 26 (1966), 273-299. MR 34 \#3295.

4. R. A. Hunt, On $L(p, q)$ spaces, Enseignement Math. (2) 12 (1966), 249-276. MR 36 \# 6921.

5. P. Krée, Interpolation d'espaces vectoriels qui ne sont ni normés, ni complets. Applications, Ann. Inst. Fourier (Grenoble) 17 (1967), fasc. 2, 137-174. MR 37 \# 4605.

6. R. O'Neil, Les fonctions conjuguées et les intégrales fractionnaires de la classe $L\left(\log ^{+} L\right)^{s}$, C. R. Acad. Sci. Paris Sér. A-B 263 (1966), A463-A466. MR 35 \#717.

7. E. M. Stein and G. Weiss, Introduction to Fourier analysis on Euclidean spaces, Princeton Univ. Press, Princeton, N.J., 1971.

8. A. Zygmund, Trigonometric series, Vols. I, II, 2nd rev. ed., Cambridge Univ. Press, New York, 1959. MR 21 \#6498.

Department of Mathematics, California institute of Technology, Pasadena, CALIFORNIA 91109 